\title{
Adição da vitamina E aos alimentos: implicações para os alimentos e para a saúde humana
}

\author{
Adding vitamin E to foods: implications for \\ the foods and for human health
}

Ellencristina da Silva BATISTA ${ }^{1}$

André Gustavo Vasconcelos COSTA'

Helena Maria PINHEIRO-SANT'ANA' ${ }^{1}$

RE S U M O

A ingestão de vitamina E em doses acima das estabelecidas pelas Dietary Reference Intake, na forma de alimentos ou suplementos, está relacionada à prevenção de doenças crônicas não transmissíveis, à estimulação do sistema imune e à modulação dos processos degenerativos relacionados ao envelhecimento. Por outro lado, a adição de vitamina $E$ aos alimentos com o intuito de alcançar tais efeitos ainda não é comum, porque não há consenso sobre a dose a ser consumida. Em muitas populações tem sido necessário ingerir alimentos fortificados com vitamina $E$, para alcançar os níveis recomendados para a ingestão adequada. Este trabalho enfoca o papel da vitamina E como um componente antioxidante utilizado pela indústria alimentícia, como um composto que exerce funções específicas no organismo humano e no alimento e como um importante nutriente que, quando adicionado aos alimentos, é capaz de atuar contra a lipoperoxidação e contribuir para o aumento de sua ingestão, além de poder reduzir os efeitos deletérios dos processos oxidativos que ocorrem no organismo humano, prevenindo as doenças crônicas não transmissíveis.

Termos de indexação: alimentos; antioxidantes; vitamina E.

A B S T R A C T

Taking vitamin $E$ in higher doses than those recommended by the Dietary Reference Intake in foods or supplements helps prevent non-communicable chronic diseases, stimulates the immune system and modulates the degenerative processes associated with aging. On the other hand, the addition of vitamin $E$ to foods to obtain those effects is not common yet because there is no consensus on an ideal dose. Vitamin $E$ has been added to the foods consumed by some populations because their intake used to be below the recommended amount. This study focuses on the role of vitamin E (1) as an antioxidant used by the food industry, (2) as a

\footnotetext{
1 Universidade Federal de Viçosa, Departamento de Nutrição e Saúde. Av. PH Rolfs, s/n., Campus Universitário, 36570-000,

Viçosa, MG, Brasil. Correspondência para/Correspondence to: H.M. PINHEIRO-SANT'ANA. E-mail: <helena.santana@ufv.br>.
} 
compound with specific functions in the human body and in foods, (3) as an important nutrient that, when added to foods, is capable of inhibiting lipoperoxidation and increase its intake and (4) as a substance that might be capable of reducing the deleterious effects of the oxidative processes that occur in the human body and therefore helping to prevent non-communicable chronic diseases.

Indexing terms: foods; antioxidants; vitamin $E$.

\section{N T R O D U Ç Ã O}

Evans \& Bishop descobriram a vitamina $\mathrm{E}$ em 1922, estudando a infertilidade em ratas, ao observarem que os animais com dieta à base de gordura suína apresentavam reabsorção fetal. Quando o germe de trigo era incluído na dieta, a síndrome de reabsorção fetal não era observada. A síndrome de reabsorção fetal foi atribuída à deficiência de um componente ativo, o qual foi denominado vitamina $E^{1}$. A manifestação de sua deficiência é rara em humanos, mas pode ocorrer em indivíduos com absorção alterada dessa vitamina ou em indivíduos com anomalias que comprometam a manutenção dos níveis corporais².

Vitamina E é a denominação genérica de oito compostos lipossolúveis, cada um dos quais com atividades biológicas específicas, sendo que o $\alpha$-tocoferol é o mais potente antioxidante ${ }^{3,4}$.

O interesse cada vez maior por essa vitamina é devido, especialmente, às funções que desempenha no organismo como agente antioxidante, envolvido no retardamento do envelhecimento e na proteção a doenças crônicas não transmissíveis, como Parkinson, Alzheimeir, câncer e doenças cardiovasculares ${ }^{3,5,6}$. A vitamina E previne o dano oxidativo celular pela inativação de radicais livres e espécies reativas de oxigênio. Atualmente, além da sua ação antioxidante, são discutidas as suas propriedades não-antioxidantes como modulação da sinalização celular e da transcrição de genes ${ }^{7}$.

Paralelamente ao efeito que a vitamina $\mathrm{E}$ exerce no organismo animal, em vegetais observa-se que é capaz de impedir a degradação da membrana celular. Essa ação antioxidante evita que os lipídios sofram lipoperoxidação e, conseqüentemente, esses vegetais apresentam vantagens nutricionais, preservando suas propriedades sensoriais ${ }^{8,9}$.
O emprego de antioxidantes sintéticos em alimentos tem sido relacionado a efeitos deletérios ao organismo humano e, por isso, desde a década de 1990 até o presente momento, tem aumentado a preocupação no sentido de obter substâncias naturais que tenham função antioxidante com a mesma eficiência dos sintéticos ${ }^{10}$. A vitamina $E$ tem sido empregada pela indústria alimentícia como um antioxidante natural, pois além dessa função é ainda um componente essencial ao organismo animal.

Este trabalho apresenta as principais funções da vitamina E no alimento, bem como as implicações sobre a saúde humana decorrentes da adição dessa vitamina aos alimentos.

\section{Análogos da vitamina $\mathrm{E}$}

Vitamina E é um termo geral empregado para designar oito compostos lipossolúveis, os $\alpha$,

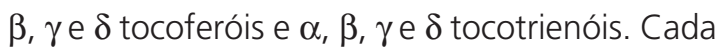
um desses compostos pode apresentar a mesma ou diferentes atividades biológicas, porém, com especificidades. Há uma diferenciação no metabolismo desses compostos, embora o processo de absorção intestinal seja o mesmo. Tal diferenciação ocorre no fígado, no qual uma proteína específica de transferência do $\alpha$-tocoferol tem maior afinidade para se ligar a esse composto na forma natural do que aos outros isômeros ou à forma sintética ${ }^{11,12}$.

O $\alpha$-tocoferol natural é constituído por um único esteroisômero, sendo designado $D-\alpha$ -tocoferol ou RRR- $\alpha$-tocoferol. A forma sintética, designada por DL- $\alpha$-tocoferol ou all-rac- $\alpha$-tocoferol, é uma mistura equimolar de 8 esteroisômeros, sendo apenas um idêntico ao natural. $A$ isomeria desses oito compostos difere devido à sua cadeia lateral, que possui centros quirais que podem 
apresentar configuração R para a direita ou S para a esquerda ${ }^{3,12-14}$. Os esteroisômeros encontrados na forma sintética são: RRR-, RRS-, RSS-, SRR-, SRS-, SSR-, SSS- ${ }^{3}$.

A adição da vitamina E em alimentos e o seu uso em formulações comerciais ou em suplementos, empregam as formas natural e sintética dessa vitamina, sendo mais comumente adicionadas na forma esterificada, como ésteres de acetato de $\alpha$-tocoferol natural ou sintético, embora também possam ser utilizadas na forma de succinato ${ }^{4,12}$. As formas esterificadas de $\alpha$-tocoferol são rotineiramente utilizadas nos suplementos de vitamina E e na fortificação de alimentos, devido à sua relativa estabilidade e ao maior tempo de vida de prateleira ${ }^{15}$.

A escolha da forma a ser utilizada tem implicações nos resultados dos estudos ${ }^{5}$, devido às distintas conseqüências sobre a saúde. As formas da vitamina $\mathrm{E}$ diferem quanto à sua atividade biológica, sendo que a vitamina $E$ natural é a que apresenta maior atividade. Na Tabela 1 estão expostos os valores da atividade biológica dos compostos comumente utilizados comercialmente $^{3}$. A atividade biológica desses compostos é expressa em Unidades Internacionais (UI), e se considera $1 \mathrm{UI}$ como $1 \mathrm{mg}$ de all rac- $\alpha$-tocoferol acetato.

A atividade biológica dos compostos descritos na Tabela 1 foi determinada em experimentos que analisaram a potencialidade de cada composto em prevenir a reabsorção fetal, em ratas com deficiência nutricional de vitamina $E^{12,14}$.

Entretanto, Burton et al. ${ }^{14}$, comparando a concentração de $\alpha$-tocoferol plasmática e tecidual em humanos, mediante suplementação da vitamina natural e sintética marcada com deutério,

Tabela 1. Atividade biológica de formas comerciais de vitamina E, expressas em UI/mg.

\begin{tabular}{lc}
\hline Composto & $\mathrm{UI} / \mathrm{mg}$ \\
\hline RRR- $\alpha$-tocoferol & 1,49 \\
Acetato de RRR- $\alpha$-tocoferila & 1,36 \\
Succinato de RRR- $\alpha$-tocoferila & 1,21 \\
All-rac- $\alpha$-tocoferol & 1,00 \\
\hline
\end{tabular}

observaram que a forma natural apresentava disponibilidade, aproximadamente, duas vezes maior que a sintética. Estudos baseados na medida da biopotência da vitamina E natural e sintética em humanos consideraram que a relação RRR: rac era de 2:1, e não mais 1,36:112. A Dietary Reference Intake (DRI) já considera a atividade biológica do $\alpha$-tocoferol sintético como 2:12.

\section{Alimentação e ingestão de vitamina $\mathrm{E}$}

A vitamina E ocorre naturalmente em alimentos de origem vegetal, principalmente nos vegetais verde-escuros, nas sementes oleaginosas, nos óleos vegetais e no germe de trigo. A ocorrência natural dos isômeros da vitamina E diferencia-se entre os vegetais ${ }^{16}$. Além de presente em alimentos vegetais, a vitamina $E$ também é encontrada em alimentos de origem animal, como gema de ovo e fígado.

Para a adequação da ingestão dos nutrientes é fundamental conhecer não somente quais alimentos são fontes de um determinado nutriente, mas também os alimentos importantes mais consumidos e que contribuem com a maior parte da ingestão da população, uma vez que a densidade do nutriente no alimento e sua freqüência de ingestão determinam as importantes fontes dietéticas $^{17,18}$.

No Brasil, ainda não foi feito nenhum estudo amplo para avaliar o consumo alimentar de vitamina E pela população. Os dados dos últimos levantamentos de disponibilidade domiciliar de alimentos, incluídos na Pesquisa de Orçamento Familiar (POF 2002-2003), mostram o consumo de alguns alimentos fontes, sem quantificar o teor de vitamina $\mathrm{E}$ ingerido ${ }^{19}$. Estudos analisados por Guinazi11 indicaram um consumo expressivo de alimentos fontes de vitamina $\mathrm{E}$, como ovos e óleos (4,3 e 7,5kg/per capita/ano, respectivamente). Entretanto, o consumo de hortaliças folhosas revelou uma média diária de apenas 7,7g, o que corresponde a 2,8kg/per capita/ano. Por outro lado, Nilson \& Piza ${ }^{20}$ relataram um baixo consumo de vitamina E pela população brasileira. 
Associadas à falta de dados do consumo alimentar dessa vitamina, as informações sobre o teor de vitamina E nos alimentos do Brasil são escassas. As informações disponíveis sobre o conteúdo de vitamina E relacionam-se a alimentos cultivados sob condições distintas daquelas existentes no Brasil ${ }^{11}$.

Nos Estados Unidos, os alimentos que fornecem o maior percentual de vitamina $E$ ingerida pelas crianças de 2 a 18 anos são margarina, o grupo dos bolos, pães e biscoitos, nozes, tomates, óleos, batata do tipo chips, leite e ovos, com uma contribuição variando de 3,9\% a 10,7\% da ingestão diária ${ }^{18}$. Para os adultos, os grupos das frutas, hortaliças e óleos contribuem cada um com 20,0\% da recomendação dessa vitamina, tomando como referência a Recommended Dietary Allowances de $1989^{21}$. Segundo Murphy et al. ${ }^{21}$, a média de ingestão da vitamina E nos Estados Unidos para homens e mulheres adultas é de 9,6 e 7,0 mg/ dia, respectivamente.

Os níveis plasmáticos de vitamina E são dependentes da ingestão de alimentos que naturalmente contêm essa vitamina, de alimentos fortificados e de suplementos de vitamina E e C ${ }^{22}$. Kang et al. ${ }^{22}$ observaram que indivíduos de ambos os sexos, que ingeriam suplementos de vitamina E ou consumiam frutas frescas ou sucos naturais, apresentavam altos níveis de $\alpha$-tocoferol plasmático. Apesar de as frutas frescas e os sucos naturais não serem fontes de vitamina $E$, seu alto conteúdo de vitamina $C$, que tem a capacidade de regenerar a vitamina $\mathrm{E}$ que foi oxidada, aumenta o nível plasmático dessa vitamina.

Na Alemanha, a ingestão adequada de vitamina $\mathrm{E}$ não era alcançada sem a ingestão de alimentos fortificados ${ }^{23-25}$. A fortificação de alimentos com vitamina $\mathrm{E}$ foi necessária para melhorar a adequação da ingestão em relação à recomendação, uma vez que os alimentos fortificados forneciam de $40 \%$ a $50 \%$ do total diário do nutriente.

Nos Estados Unidos, Booth et al. ${ }^{17}$ também observaram que a ingestão de vitamina $\mathrm{E}$ não atingia a cota recomendada pela RDA de 1989, de 8 e 10mg/dia para mulheres e homens, respectivamente, e que o teor de vitamina E proveniente de alimentos fortificados correspondia a um quinto da ingestão total.

Do mesmo modo que em outros países, a baixa ingestão de vitamina $E$ foi observada em homens cubanos saudáveis, que apresentavam níveis plasmáticos baixos dessa vitamina ${ }^{26}$. A baixa ingestão de vitamina $\mathrm{E}$ também foi observada em adolescentes suíços ${ }^{27}$.

Contudo, a nova recomendação de vitamina $E$, de acordo com as DRI estabelecidas em 2000, pelo Instituto de Medicina dos Estados Unidos, preconiza uma ingestão de $15 \mathrm{mg} / \mathrm{dia}$, a qual vem sendo questionada uma vez que é maior que a ingestão alimentar de vitamina E obtida pela maioria dos americanos ${ }^{28}$. Essa nova recomendação também preconiza que, dos oito isômeros da vitamina $\mathrm{E}$, apenas o $\alpha$-tocoferol deve ser considerado, por manter sua concentração plasmática ${ }^{29,30}$. O $\gamma$-tocoferol é um importante isômero da vitamina E na alimentação e sua concentração plasmática vem sendo inversamente relacionada ao risco de desenvolvimento de doenças cardiovasculares e câncer de próstata ${ }^{31}$. Além disso, a ingestão de $\gamma$-tocoferol vem aumentando devido ao aumento da ingestão de óleo de soja, rico nesse composto, em detrimento do consumo de alimentos fontes de $\alpha$-tocofero ${ }^{30}$.

Para alcançar a atual recomendação (DRI, 2000) é necessário ingerir grande quantidade de alimentos ricos em ácidos graxos insaturados, o que, conseqüentemente, aumentará a necessidade de vitamina E para prevenir a oxidação ${ }^{28}$. Uma dieta rica em frutas e hortaliças e reduzida em gorduras, provavelmente, contém menos de $15 \mathrm{mg}$ de $\alpha$-tocoferol, a não ser que haja aumento da ingestão de óleos, nozes e cereais integrais ${ }^{30}$.

Vale ressaltar que as DRI para essa vitamina são determinadas como a quantidade necessária para evitar a sua deficiência, e não levam em consideração os seus efeitos antioxidantes. Entretanto, Guinazi ${ }^{11}$ relata que a atual recomen-dação levaria em consideração a ação da vitamina E na prevenção de doenças relacionadas ao estresse 
oxidativo. Vários estudos têm descrito que os efeitos da ingestão de doses de vitamina $\mathrm{E}$ acima das estabelecidas pela atual recomendação, na forma de alimentos ou suplementos, atuam na prevenção de doenças crônicas não transmissíveis, na estimulação do sistema imune e na modulação dos processos degenerativos relacionados ao envelhecimento ${ }^{5,6}$.

\section{Ação antioxidante da vitamina $E$}

O processo oxidativo nos alimentos pode ser inibido pela adição de nitrato, metais quelantes e substâncias com propriedades antioxidantes. Entre essas substâncias destacam-se a vitamina $E$, a vitamina $C$ e o $\beta$-caroteno ${ }^{9}$.

No processo de deterioração, as membranas celulares são as estruturas mais acometidas pela peroxidação lipídica. Esse processo provoca alterações estruturais e, conseqüentemente, sua permeabilidade, o que acarreta perdas da seletividade na troca iônica, liberação de compostos citoplasmáticos e formação de produtos tóxicos ${ }^{8}$.

O processo de oxidação em alimentos cárneos inicia-se na fração fosfolipídica das membranas celulares. A oxidação lipídica é a maior causa de deterioração e perda de qualidade de produtos, ocasionando alterações no sabor, no aroma, na cor, na textura, no valor nutricional e no que condiz à segurança microbiológica?.

A função antioxidante da vitamina $E$ tem sido amplamente estudada, uma vez que faz parte de um sistema de proteção que envolve outros componentes, como o ácido ascórbico e o selênio ${ }^{3}$.

Por ser um composto lipossolúvel e compor as membranas celulares, a vitamina E é capaz de impedir a deterioração lipídica e, conseqüentemente, impedir a formação de hidroperóxidos, devido à sua capacidade antioxidante ${ }^{9}$. A capacidade de essa vitamina impedir a oxidação depende de sua concentração no tecido.

Ferreira \& Matsubara ${ }^{8}$, descrevem que a lipoperoxidação é uma reação em cadeia, caracte- rizada pelas etapas de iniciação, propagação e terminação (Figura 1).

Segundo Bianchini \& Penteado ${ }^{3}$, a reação de iniciação é muito lenta e depende da presença de catalisador que pode ser luz, calor, metais ou enzimas.

A vitamina E é considerada um potente antioxidante, reagindo 200 vezes mais rápido contra radicais peroxila $(\mathrm{LOO} \bullet)$ em relação a antioxidantes sintéticos ${ }^{3}$.

Para proteger as células do processo de oxidação contra agentes óxido-redutores, produzidos de forma endógena ou sob a ação de substâncias exógenas, a célula possui um sistema de defesa, que pode atuar em duas linhas: a primeira atua como detoxificadora do agente antes que cause a lesão, a qual é constituída por glutationa reduzida, superóxido dismutase, catalase, glutationa peroxidase e vitamina $\mathrm{E}$; a segunda forma de defesa tem função de reparar a lesão ocorrida, sendo constituída pelo ácido ascórbico, pela glutationa peroxidase e pela glutationa-redutase.

A vitamina E apresenta-se como componente estrutural da membrana e a maior parte

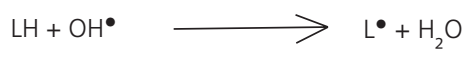

Iniciação: Seqüestro do hidrogênio do ácido graxo poliinsaturado da membrana celular

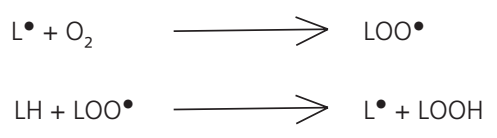

Propagação: o $\mathrm{L}^{\bullet}$ reage com o $\mathrm{O}_{2}$, resultado em LOO . Este novamente seqüestra hidrogênio do ácido poliinsaturado, gerando o segundo $L^{\bullet}$

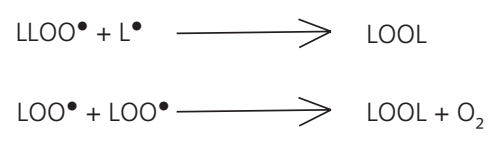

Terminação: autodestruição de radicais formados na etapa de Programação.

Figura 1. Etapas da reação de lipoperoxidação. 
dos demais agentes antioxidantes encontra-se no meio intracelular ${ }^{8}$. A presença da vitamina $E$ na membrana é de extrema importância, pois exerce um efeito protetor contra a degradação lipídica e, conseqüentemente, contra o extravasamento de material intracelular, que comprometeria o funcionamento do organismo.

\section{Estabilidade oxidativa da vitamina E nos alimentos}

A estabilidade oxidativa, ou seja, a capacidade de um composto em não oxidar, é de grande importância para a garantia da qualidade dos alimentos. Alguns compostos presentes nos alimentos apresentam ação pró-oxidante e outros apresentam ação antioxidante, podendo ser intrínsecos ou adicionados aos alimentos. Além disso, componentes externos, como luz, temperatura e tempo de estocagem, também podem interferir acelerando o processo de deterioração dos produtos. Dessa forma, a vitamina E pode ser adicionada aos alimentos com o objetivo de evitar o processo oxidativo.

Stapelfeldt et al. ${ }^{32}$ investigaram a incorporação e retenção de vitamina $\mathrm{E}$ no leite de vaca, após a injeção intraperitoneal de $10 \mathrm{~g}$ de all-rac- $\alpha$-tocoferol acetato no plasma. Os resultados demonstraram que, quanto maior o conteúdo de $\alpha$-tocoferol, menor foi a concentração de radicais livres no leite.

Em um estudo com pães foram adicionados 200, 400, 800 e 1.600 IU de vitamina E por unidade de $50 \mathrm{~g}$ de pão francês, na forma de $\alpha$-tocoferil acetato. Os teores de vitamina $\mathrm{E}$ foram analisados nos pães frescos e após sete dias de estocagem. Os níveis adicionados não exerceram nenhum efeito adverso na qualidade do pão. Entretanto, somente cerca de dois terços da vitamina adicionada foi retida nos pães, sendo o restante perdido durante o processamento ${ }^{33}$. Nos pães frescos, os valores da retenção da vitamina E foram semelhantes (média de 67,2\%) em todos os níveis da vitamina $E$ testados, sugerindo que não houve perdas significativas de vitamina em relação ao período de estocagem e que a adição de 800 Ul em pães de $50 \mathrm{~g}$ seria suficiente para fornecer os níveis diários requeridos. Nesse estudo concluiu-se que a vitamina $\mathrm{E}$ pode ser adicionada ao pão em níveis relativamente elevados, sem ocasionar efeitos adversos às características sensoriais.

Em um estudo sobre o efeito da vitamina E na sua forma de $\alpha$-tocoferol sobre a oxidação lipídica de carne bovina, a porcentagem de oxigênio atmosférico foi alterada para níveis de $20 \%$, $40 \%, 60 \%$ ou $80 \%$ de $\mathrm{O}_{2}$, adicionando ou não $\alpha$-tocoferol. Sem a adição de $\alpha$-tocoferol, não houve diferença no conteúdo de oximioglobina das peças das carnes estocadas até 4 dias com as modificações atmosféricas. Após o $7^{\circ}$ dia, houve redução $(p<0,05)$ dos níveis de oximioglobina e dos níveis de oxigênio. A oxidação lipídica das peças aumentou significativamente entre 7 e 10 dias de estocagem, quando o conteúdo de oxigênio atmosférico foi modificado em 40\%, 60\% e $80 \%$. A adição de $\alpha$-tocoferol foi de 300 e $3000 \mathrm{mg} / \mathrm{kg}$ de lipídio, tendo como veículo azeite de oliva. A adição de $\alpha$-tocoferol exógeno conduziu a uma redução $(p<0,05)$ da oxidação lipídica em peças bovinas estocadas em altas atmosferas de $\mathrm{O}_{2}$, mas não foi observado efeito positivo com a adição de $\alpha$-tocoferol quando as peças foram submetidas a baixas pressões ${ }^{34}$.

Nenadis et al. ${ }^{35}$ pesquisaram a atividade dos antioxidantes ácido 6-hidroxi-2, 5,7,8-tetrametilcromo-2-carboxílico (Trolox ${ }^{\circledR}$ ), butilhidroxianisol (BHA), butilhidroxitolueno (BHT), terc-butil-hidroquinona (TBHQ), $\alpha$-tocoferol e ácido caféico, sob fosfatidilcolina de lipossomas. Os resultados demonstraram que o $\alpha$-tocoferol apresentou uma média potência em relação aos outros componentes testados. O BHT e o BHA apresentaram os melhores efeitos antioxidantes. Passotto et al. ${ }^{10}$ alertaram que esses compostos podem causar efeitos adversos ao organismo animal como, por exemplo, hemorragia e extensa proliferação de células no pulmão. Devido a tais inconvenientes, nos últimos anos tem havido a preocupação de empregar substâncias naturais com a mesma função e eficiência dos antioxidantes sintéticos. 
Albalá-Hurtado et al. ${ }^{36}$ investigaram a estabilidade de vitaminas hidrossolúveis do complexo $B$ e das vitaminas lipossolúveis $A$ e $E$ em 3 tipos de leites infantis líquidos, após 12 meses de estocagem em temperaturas de 20,30 e $37^{\circ} \mathrm{C}$. Os teores de vitamina $A$ reduziram quando a temperatura foi de $37^{\circ} \mathrm{C}$. Por outro lado, os níveis de vitaminas hidrossolúveis e de vitamina E permaneceram constantes durante a estocagem nas 3 temperaturas testadas, demonstrando maior estabilidade.

Miquel et al. ${ }^{37}$ investigaram os efeitos da estocagem ( 0 a 17 meses) e da temperatura (22 e $37^{\circ} \mathrm{C}$ ) sobre $\alpha$-tocoferol acetato, $\alpha$, $\gamma$ e $\delta$-tocoferol em fórmulas infantis. Os teores dos isômeros de vitamina E adicionados, analisados por cromatografia líquida de alta eficiência (CLAE) utilizando detecção por fluorescência, foram iguais para todos os produtos testados. Os resultados demonstraram que os teores de $\alpha$, $\gamma$ e $\delta$-tocoferol adicionados reduziram tanto com a estocagem quanto com a variação de temperatura. A temperatura de $37^{\circ} \mathrm{C}$ foi a que levou às maiores perdas. Todos os compostos avaliados apresentaram papel antioxidante, contribuindo para a estabilidade da porção lipídica. Os níveis de $\alpha$-tocoferol acetato não foram afetados pela temperatura ou período de estocagem, colocando em dúvida seu papel como um antioxidante.

De acordo com Flachowsky et al..$^{38}$, a suplementação de rações para tratamentos de animais de abate, como aves, é uma estratégia eficaz para aumentar a ingestão de vitamina $\mathrm{E}$ pelos consumidores de aves. Nesse estudo, as concentrações de tocoferol na carne aumentaram com a suplementação de vitamina E. No entanto, os autores relataram que a suplementação depende do custo de vitamina $E$ adicionada e do benefício financeiro gerado aos produtores.

\section{Implicaçõos da adição de vitamina E aos alimentos para a saúde humana}

Além de ocorrer naturalmente nos alimentos, a vitamina E pode ser adicionada para prevenir a oxidação dos alimentos e, também, com o intuito de aumentar o teor dessa vitamina, ou seja, fortificá-los. Vale ressaltar que o aumento do conteúdo de vitamina E no alimento pode ser feito, também, por meio da adição dessa vitamina à dieta de animais domésticos aumentando, conseqüentemente, o seu conteúdo nos alimentos derivados desses animais ${ }^{38}$.

Estudos epidemiológicos e clínicos vêm evidenciando a eficácia da ingestão de doses de vitamina $\mathrm{E}$ acima das recomendações na prevenção e no tratamento de doenças. Contudo, os resultados ainda são inconsistentes e a dose supra nutricional que leva aos efeitos desejados ainda não foi estabelecida, embora se suponha que a dose necessária para alcançar tais efeitos seja muito maior que a recomendação da DRI de $2000^{6}$.

Segundo Schwenke ${ }^{39}$, os estudos populacionais sugerem que os níveis de vitamina $E$ capazes de reduzir o risco de doença cardiovascular podem ser obtidos apenas por suplementação.

Os estudos de fortificação alimentar ressaltam a importância da fortificação com nutrientes, especialmente ferro, vitamina A e iodo, que estão relacionados a carências nutricionais que acometem grande parte da população mundial, principalmente nos países em desenvolvimento e subdesenvolvidos 20,40,41. A fortificação de alimentos com vitamina $\mathrm{E}$, bem como com outros nutrientes relacionados à prevenção e ao controle das doenças crônicas não transmissíveis, ainda não é comum, até mesmo porque não há consenso sobre a dose a ser consumida para alcançar tais efeitos. Entretanto, em muitas populações é necessário ingerir alimentos fortificados com vitamina E para alcançar a sua recomendação 17,23-25.

A necessidade de alta ingestão contínua de vitamina $E$, via suplementação com cápsulas, vem sendo estudada na prevenção do estresse oxidativo. Entretanto, além da dose de vitamina E ingerida devem-se considerar as diferenças dos seus vitâmeros, no que condiz à biopotência e ao metabolismo ${ }^{3}$. 
Hayes et al. ${ }^{42}$ observaram que a adição de vitamina E na forma de all-rac- $\alpha$-tocoferil-acetato em níveis de 100 ou $200 \mathrm{mg} / 960 \mathrm{~mL}$ de leite consumido diariamente, levou ao aumento no teor dessa vitamina nas lipoproteínas plasmáticas dos indivíduos, efeito que não foi observado na dose de $30 \mathrm{mg}$. Esse resultado pode ter implicações para a saúde da população, devido aos efeitos benéficos da alta concentração plasmática de $\alpha$ -tocoferol, relacionados à prevenção das doenças cardiovasculares, de certos tipos de câncer e de outras doenças relacionadas ao estresse oxidativo ${ }^{42}$.

O consumo de 15g/dia de margarina fortificada com $121 \mathrm{mg}$ de vitamina $C ; 31 \mathrm{mg}$ de vitamina $E, 2,7 \mathrm{mg}$ de $\alpha$-caroteno e 5,3mg de $\beta$-caroteno, incluídos em uma dieta normal, durante quatro semanas, levou ao aumento dos níveis plasmáticos desses antioxidantes, bem como à maior resistência da LDL à oxidação ${ }^{43}$.

Dados do padrão alimentar nos Estados Unidos, de 1987 a 1992, mostraram que a ingestão de cereais matinais fortificados contribuiu com a ingestão de vários nutrientes entre eles a vitamina $\mathrm{E}^{44}$.

Serra-Manjem ${ }^{45}$ verificou que a maior parte das crianças e adolescentes na Europa apresentava alto risco de desenvolver deficiências nutricionais de alguns minerais e vitaminas, entre estas a de vitamina $E$, e sugeriu que os cereais matinais fortificados podem contribuir significativamente com a ingestão de micronutrientes.

Segundo Meydani ${ }^{6}$, a ingestão de alimentos contendo alta quantidade de vitamina E é recomendada para idosos. Além disso, esse autor relata que o consumo de refeições diárias para atingir a dose de 60 UI (90mg) dessa vitamina conduziu a efeitos benéficos no sistema imune. Entretanto, para alcançar essa dose seria necessário ingerir alimentos ricos em ácidos graxos insaturados, o que aumentaria a necessidade de vitamina E para prevenir a oxidação. Portanto, a ingestão desse nível seria mais benéfica por meio da fortificação de alimentos com vitamina $\mathrm{E}$.
A adição da vitamina $E$ deve ser feita, preferencialmente, nos alimentos que possuem maior teor de gordura, como o leite e a margarina ou os que são comumente ingeridos com alimentos ricos em gordura como pão e cereal matinal, pois a eficiência na absorção dessa vitamina é aumentada pelo consumo concomitante de gordura na dieta ${ }^{3,46}$, levando a um aumento da biopotência. Devido à sua ação antioxidante, a vitamina $E$ pode ainda prevenir a oxidação dos ácidos graxos poliinsaturados, impedindo a oxidação do próprio alimento.

A fortificação de alimentos focaliza os nutrientes com baixos níveis de ingestão. Entretanto, para avaliar a importância da ingestão de baixos níveis de vitamina $\mathrm{E}$, é necessário comparar com níveis de colesterolemia e não há dados disponíveis a respeito do risco de doenças crônicas e diferentes níveis de vitamina $\mathrm{E}^{47}$.

A Vigilância Sanitária, que regulamenta a adição de nutrientes em alimentos no Brasil, define que para a fortificação ou o enriquecimento de vitaminas e de minerais, $100 \mathrm{~mL}$ ou $100 \mathrm{~g}$ do produto pronto para o consumo forneça, no mínimo, $15 \%$ da DRI de referência, no caso de líquidos, e $30 \%$ da DRI de referência, no caso de sólidos. Para alimentos suplementados a legislação estabelece no mínimo 25\% e no máximo 100\% da DRI do nutriente suplementado ${ }^{48,49}$.

O estado nutricional de vitamina E está relacionado ao desenvolvimento e à progressão das doenças crônicas não transmissíveis. Se indivíduos com risco para desenvolvimento de tais doenças apresentarem deficiência nutricional de vitamina E esse risco torna-se maior e, quando a doença já está instalada, pode gerar um quadro mais grave ${ }^{49}$. Não obstante, a fortificação alimentar com vitamina $\mathrm{E}$ pode levar a aumentos significantes na ingestão desse nutriente ${ }^{50}$.

\section{ONCLUS Ã O}

A ação antioxidante da vitamina E envolve a inibição da lipoperoxidação das membranas celulares, impedindo assim a deterioração de 
ácidos graxos indispensáveis para o organismo. Devido à sua capacidade antioxidante, além dos benefícios para a saúde, a vitamina $\mathrm{E}$ tem ações benéficas sobre o alimento, minimizando a formação de radicais livres.

Embora a deficiência de vitamina $E$ não seja um problema de saúde pública, a ingestão de doses maiores que a estabelecida pela atual recomendação, tem conduzido a efeitos benéficos contra as doenças causadas pelo estresse oxidativo, o que sugere que a fortificação dos alimentos pode contribuir para a ingestão adequada de vitamina $\mathrm{E}$.

Os alimentos que possuem alto teor de gordura, como o leite e a margarina, ou aqueles que são comumente ingeridos com outros alimentos ricos em gordura, podem ser, preferencialmente, fortificados com vitamina E, por propiciarem maior absorção desse nutriente.

A vitamina $E$ apresenta efeitos positivos, tanto para a saúde humana quanto para a qualidade nutricional dos alimentos, e a sua utilização na indústria alimentícia como antioxidante parece ser uma estratégia eficaz para o aumento da ingestão diária desse micronutriente.

Apesar de sua importância, ainda são escassos no Brasil dados sobre o consumo alimentar pela população e estado nutricional e o conteúdo de vitamina $E$ natural nos alimentos produzidos no País, evidenciando a importância e a necessidade de estudos relacionados a essa vitamina.

\section{COLABORADORES}

E.S. BATISTA e A.G.V. COSTA participaram do levantamento bibliográfico, concepção e redação do artigo. H.M. PINHEIRO-SANT'ANA participou da orientação e redação do artigo.

\section{REFERÊ N CIAS}

1. Traber MG. Vitamin E. In: Shills ME, Olson JA, Shike $M$, Ross AC. Modern nutriton in health and disease. New York: Lea \& Febicer; 1994. p.326-41.
2. Institute of Medicine. Dietary intake for vitamin C, vitamin E, selenium e carotenoids. Washington (DC): National Academic Press; 2000 [cited 2005 Jun 10] Available from: htttp://www.nap.edu/ openbook/03306935/htm

3. Bianchini R, Penteado MVC. Vitamina E. In: Vitaminas: aspectos nutricionais, bioquímicos, clínicos e analíticos. Barueri: Manole; 2003. p.23-164.

4. Ball GFM. Vitamin E. In: Bioavailability and analysis of vitamin in foods. London: Chapman \& Hall; 1998. p.195-239.

5. Brigelius-Flohé R, Kelly FJ, Salonen JT, Neuzil J, Zingg JM, Azzi A. The European perspective on vitamin $\mathrm{E}$ : current knowledge and future research. Am J Clin Nutr. 2002; 76(4):703-16.

6. Meydani M. Effect of functional food ingredients: vitamin $\mathrm{E}$ modulation of cardiovascular diseases and immune status in the elderly. Am J Clin Nutr. 2000; 71(6 Suppl):1665S0-68S.

7. Munteanu A, Zingg JM, Azzi A. Anti-atherosclerotic effects of vitamin E: myth or reality. J Cell Mol Med. 2004; 8(1):59-76.

8. Ferreira ALA, Matsubara LS. Radicais livres: conceitos, doenças relacionadas, sistema de defesa estresse oxidativo. Rev Ass Med Brasil. 1997; 43(1):61-8.

9. Buckley DJ, Morrissey PA, Gray Jl. Influence of dietary vitamin $\mathrm{E}$ on the oxidative stability and quality of pig meat. J Anim Sci. 1995; 73(10): 3122-30.

10. Passotto JA, Penteado MVC, Mancini-Filho J. Atividade antioxidante do $\beta$-caroteno e da vitamina A. Estudo comparativo com antioxidante sintético. Cienc Tecnol Aliment. 1998; 18(1):68-72.

11. Guinazi M. Tocoferóis e tocotrienóis em hortaliças, ovos e óleos vegetais utilizados em restaurantes comerciais [dissertação]. Viçosa: Universidade Federal de Viçosa; 2004.

12. Hope PP, Krennrich G. Biovailability and potency of natural-source and all-racemic $\alpha$-tocopherol in the human: a dispute. Eur J Nutr. 2000; 39(5): 183-93.

13. Stone WL, LeClair I, Ponder T, Baggs G, Reis BB. Infants discriminate between natural and synthetic vitamin E. Am J Clin Nutr. 2003; 77(4):899-906.

14. Burton GW, Traber MG, Acuff RV, Walters DN, Kayden $H$, Hughes $L L$, et al. Human plasma and tissue a-tocopherol concentrations in response to supplementation with deuterated natural and synthetic vitamin E. Am J Clin Nutr. 1998; 64(4): 669-84.

15. Trumbo PR, Yates AA, Schlicker-Renfro S, Suitor $C$. Dietary Reference Intakes: revised nutritional 
equivalents for folate, vitamin $\mathrm{E}$ and provitamin $\mathrm{A}$ carotenoids. J Food Compos Anal. 2003; 16: 379-82.

16. Setiadia DH. Vitamin E models. Shortened side chain models of $a, b, g$ and $d$ tocopherol and tocotrienol: a density functional study. J Mol Struct. 2003; 637:11-26.

17. Booth SL, Tucker KL, McKeown NM, Davidson KW, Dallal GE, Sadowski JA. Relationships between Dietary Intakes and fasting plasma concentrations of fat-soluble vitamins in humans. J Nutr. 1997; 127(4):587-92.

18. Subar AF, Krebs-Smith SM, Cook A, Kahle LL. Dietary sources of nutrients among US children, 1989-1991. Pediatrics. 1998; 102(4 PT1):913-23.

19. Instituto Brasileiro de Geografia e Estatística. Pesquisa de orçamentos familiares - POF 2002-2003. Rio de Janeiro; 2004.

20. Nilson A, Piza J. Food fortificaton: a toll for fighting hidden hunger. Food Nutr Bull. 1998; 19(1):49-60.

21. Murphy SP, Subar AF, Block G. Vitamin E intakes and sources in the United States. Am J Clin Nutr. 1990; 52(2):361-7.

22. Kang MJ, Lin YC, Yeh WH, Wen-Harn P. Vitamin E status and its dietary determinants in Taiwanese: results of the Nutrition and Health Survey in Taiwan 1993-1996. Eur J Nutr. 2004; 43(2):86-92.

23. Sichert-Hellert W, Kersting M, Manz F. Changes in time-trends of nutrient intake from fortified and non-fortified food in German children and adolescents: 15 year results of the DONALD Study. Eur J Nutr. 2001; 40(2):49-55.

24. Sichert-Hellert W, Kersting M, Alexy U, Manz F. Tenyear trends in vitamin and mineral intake from fortified food in German children and adolescents. Eur J Clin Nutr. 2000; 54(1):81-6.

25. Beitz R, Mensink GBM, Fischer B, Thamm M. Vitamins dietary intake and intake from dietary supplements in Germany. Eur J Clin Nutr. 2002; 56(6):539-45.

26. Arnaud J, Fleits $P$, Chassagne $M$, Verdura T, Barnovin J, Chacomac JP, et al. Seasonal variations of antioxidant imbalance in Cuban healthy men. Eur J Clin Nutr. 2001; 55(1):29-38.

27. Decarli B, Cavadini C, Grin J, Blondel-Lubrano A, Narring F, Michaud PA. Food and nutrient intakes in a group of 11 to 16 year old swiss teenagers. Int J Vitam Nutr Res. 2000; 70(3):139-47

28. Horwitt MK. Critique of the requirement for vitamin E. Am J Clin Nutr. 2001; 73(6):1003-5.

29. Murphy SP. Dietary References Intakes of the U.S. and Canada: update on implications for nutrients databases. J Food Compos Anal. 2002; 5:411-7.
30. Traber MG. Vitamin E: too much or not enough? Am J Clin Nutr. 2001; 73(6):987-98.

31. Jiang Q, Christen S, Shigenaga MK, Ames BN. Tocopherol, the major form of vitamin $\mathrm{E}$ in the US diet, deserves more attention. Am J Clin Nutr. 2001; 74(6):714-22.

32. Stapelfeldt H, Nielsen K N, Jensen S K, Skibsted L $\mathrm{H}$. Free radical formation in freeze-dried raw milk in relation to its $\alpha$-tocopherol level. J Dairy Res. 1999; 66(3):461-6.

33. Ranhotra GS, Gelroth JA, Okot-Kotber BM. Stability and dietary contribution of vitamin $\mathrm{E}$ added to bread. Cereal Chem. 2000; 77(2):159-62.

34. O'Grady MN, Monahan FJ, Burke RM, Allen P. The effect of oxygen level and exogenous $\alpha$-tocopherol on the oxidative stability of minced beef in modified atmosphere packs. Meat Sci. 2000; 55(1): 39-45.

35. Nenadis N, Zafiropoulou I, Tsimidou M. Commonly used food antioxidants: a comparative study in dispersed systems. Food Chem. 2003; 82(3): 403-7.

36. Albalá-Hurtado S, Veciana-Nogués MT, VidalCarou1 MC, Mariné-Font A. Stability of vitamins during the storage of liquid infant milks. J Dairy Res. 2000; 67(2):225-31.

37. Miquel E, Alegría A, Barberá R, Farré R, Clemente G. Stability of tocopherols in adapted milk-based infant formulas during storage. Int Dairy J. 2004; 14:1003-11.

38. Flachowsky G, Engelman D, Sünder A, Halle I, Sallmann HP. Eggs and poultry meat as tocopherol sources in dependence on tocopherol supplementation of poultry diets. Food Res Int. 2002; 35:239-43.

39. Schwenke DC. Antioxidants and atherogenesis. J Nutr Biochem. 1998; 9:424-45.

40. Lutter CK, Dewey KC. Proposed nutrient composition for fortified complementary foods. J Nutr. 2003; 133(9):3011S-20S.

41. Allen LH. Interventions for micronutrient deficiency control in developing countries: Past, present and future. J Nutr. 2003; 133(11 Suppl 2):3874S-8S.

42. Hayes KC, Pronczuk A, Perlman D. Vitamin E in fortified cow milk uniquely enriches human plasma Lipoproteins. Am J Clin Nutr. 2001; 74(2): 211-8.

43. Van Het Hof KH, Tijburg LBM, Boer HSM , Wiseman SA, Weststrate JA. Antioxidant fortified margarine increases the antioxidant status. Eur J Clin Nutr. 1998; 52(4):292-2

44. Norris J, Harnack L, Carmichael S, Pouane T, Wakimoto $\mathrm{P}$, Block G. US trends in nutrient intake: 
the 1987 and 1992 national health interview surveys. Am J Public Health. 1997; 87(5):740-6.

45. Serra-Majem L. Vitamin and mineral intake in European children. Public Health Nutr. 2001; 4(1A):101-7.

46. Iuliano L, Micheletta F, Maranghi M, Frati G, Diczfalusy U, Violi F. Biovailability of Vitamin E as function of food intake in healthy subjects - effects on plasma peroxide-scavenging activity and cholesterol-oxidation products. Arterioscler Thromb Vasc Biol. 2001; 21(10):E34-7.

47. Tomkins A. Vitamin and mineral nutrition for the health and development of the children of Europe. Public Health Nutr. 2001; 4(1A):91-9.

48. Brasil. Portaria $n^{\circ} 31$, de 13 de janeiro de 1998. Alimentos adicionados de nutrientes essenciais: alimentos enriquecidos [acesso em 10 jun 2005]. Disponível em: http://www.anvisa.gov.br/legis/ index.htm

49. Brasil. Portaria n 32, de 13 de janeiro de 1998. Alimentos adicionados de nutrientes essenciais: suplementos vitamínicos e ou minerais [acesso em 10 jun 2005]. Disponível em: http://www.anvisa. gov.br/legis/index.htm

50. Landvik S. Vitamin E from supplements has good bioavailability. Am J Clin Nutr. 2004; 80(3):784-5.

Recebido em: 18/7/2005

Versão final reapresentada em: 14/2/2007

Aprovado em: 14/3/2007 
